\title{
Erratum: DPA Calculation for the D-D Nuclear Fusion Reaction in the KSTAR PFC
}

\author{
[J. Korean Phys. Soc. 60, 773 (2012)] \\ Bo-Young LEe, Joo-Hee OH and Seung-Kook Ko* \\ Department of Physics, University of Ulsan, Ulsan 680-749, Korea \\ Hee-Seock LEE \\ Pohang Accelerator Laboratory, POSTECH, Pohang 790-784, Korea
}

DOI: $10.3938 / \mathrm{jkps} .60 .1993$

The acknowledgement should be corrected to 'This work was supported by the National Research Foundation of Korea (NRF) grant funded by the Korea government (MEST) (2011-0018740).'. 